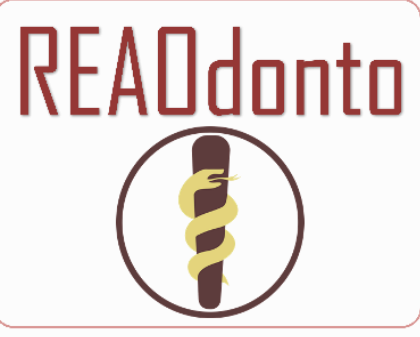

Revista Eletrônica Acervo
Odontológico
RELATO DE CASO

Recebido em: 6/2020

Aceito em: 7/2020

Publicado em: 8/2020

\title{
Comprometimento de papila parotídea em lesão de hiperplasia fibrosa inflamatória: relato de caso
}

\author{
Commitment of parotid paper in lesion of inflammatory fibrous hyperplasia: case report
}

Compromiso del papel de parotid en lesión de hiperplasia fibrosa inflamatoria: reporte de caso

Luan Gabriel Marques Canali ${ }^{1}$, Camila Pereira de Jesus ${ }^{1}$, Eliana de Souza Bastos Mazuqueli Pereira $^{1}$, Percyleine Pelegrini Herculiani ${ }^{1}$, Amanda Pelegrini Herculiani ${ }^{2}$, Rachel Gomes Eleutério $^{1 *}$.

Resumo: Este artigo buscou relatar a realização da remoção de uma lesão de hiperplasia fibrosa inflamatória (HFI) localizada em mucosa jugal, sobre região de papila parotídea. Para isso, o indivíduo C.A.C.C., sexo masculino, 56 anos, procurou atendimento na Clínica de Estomatologia do curso de Odontologia da Universidade de Marília (UNIMAR), tendo como queixa principal a sensação de um leve incômodo ao realizar movimentos de fala e mastigação. Então, prosseguiu-se o atendimento com o exame clínico do mesmo e, posteriormente, procedimento cirúrgico de biópsia excisional da massa hiperplásica. Em seguida, a lesão foi submetida ao exame anatomopatológico, com finalidade de confirmação do diagnóstico inicial. Conclui-se, então, ser de extrema importância a promoção da terapêutica adequada ao paciente frente as suas necessidades, uma vez que há obtenção de qualidade de vida, além do descarte da presença de lesões de caráter maligno que possam acometer de forma mais severa o indivíduo.

Palavras-chave: Hiperplasia, Mucosa bucal, Região parotídea.

\begin{abstract}
This article seeks to relate the removal of an inflammatory fibrous hyperplasia (HFI) lesion located in the jugal mucosa, over the parotid papilla region. For this, the individual C.A.C.C., male, 56 years old, sought care at the Stomatology Clinic of the Dentistry Course of the University of Marília (UNIMAR), having as main task the presence of a slight discomfort when performing speech and chewing movements. Then, continue the service with the clinical examination of the same and, later, the surgical procedure of excisional biopsy of the hyperplastic mass. Then, a lesion was submitted to anatomopathological examination, with permission to confirm the initial diagnosis. In conclusion, then, it is extremely important to promote appropriate therapy for the patient in face of their needs, since there is use of quality of life, in addition to discarding the presence of malignant lesions that can affect more severely or individually.
\end{abstract}

Keywords: Hyperplasia, Mouth mucosa, Parotid region.

1 Universidade de Marília (UNIMAR). Marília - São Paulo. *E-mail: rachel.ge@hotmail.com

${ }^{2}$ Laboratório LOGOS. Londrina - Paraná. 
Resumen: Este artículo busca relacionar la extirpación de una lesión inflamatoria de hiperplasia fibrosa (HFI) ubicada en la mucosa yugal, sobre la región de la papila parótida. Para ello, el individuo C.A.C.C., hombre, 56 años, buscó atención en la Clínica de Estomatología del Curso de Odontología de la Universidad de Marília (UNIMAR), teniendo como tarea principal la presencia de una leve molestia al realizar movimientos de habla y masticación. Luego, continúe el servicio con el examen clínico de la misma y, más tarde, el procedimiento quirúrgico de biopsia por escisión de la masa hiperplásica. Luego, se sometió una lesión a examen anatomopatológico, con permiso para confirmar el diagnóstico inicial. En conclusión, entonces, es extremadamente importante promover una terapia adecuada para el paciente frente a sus necesidades, ya que se utiliza la calidad de vida, además de descartar la presencia de lesiones malignas que pueden afectar más severamente o individualmente.

Palabras clave: Hiperplasia, Mucosa bucal, Región parotídea.

\section{INTRODUÇÃO}

A hiperplasia fibrosa inflamatória (HFI) baseia-se em uma lesão de origem reativa, originada por meio de estímulos crônicos de baixa intensidade produzidos sobre a mucosa oral. Desse modo, o termo hiperplasia refere-se ao aumento de número de células presentes em um determinado tecido, resultantes de uma resposta acentuada de reparação tecidual (DUTRA KL, et al., 2018). Ademais, tal lesão pode, ainda, ser classificada como um processo proliferativo não neoplásico (PPNN), uma vez que ocorre a proliferação tecidual por meio de processos inflamatórios (MENDES JK, et al., 2019).

Clinicamente, a hiperplasia fibrosa inflamatória pode apresentar-se como um processo exofítico ou como uma placa bem definida, variando de firme a flácida quando submetida à palpação. A base pode ser séssil ou pediculada, com coloração semelhante à mucosa ou eritematosa, de crescimento lento, sendo, geralmente, assintomática. Estudos revelam que traumas mastigatórios fazem com que seu local de maior acometimento intraoral seja ao longo da linha oclusal da mucosa jugal, seguido pelo tecido gengival (BINITA G, et al., 2016; RAMU S e RODRIGUES C, 2012; SRIVASTAVA KC, 2019).

Farynowska J, et al. (2018) afirmaram que a lesão decorre de um fator irritante crônico, como biofilme, cálculo, aparelho ortodôntico, prótese mal adaptada, má-oclusão ou desequilíbrios hormonais que estimulam a formação de um tecido de granulação e, posteriormente, o processo de fibrose. Além disso, sua etiologia pode estar relacionada a um transtorno de comportamento repetitivo focado no corpo, tal como 0 hábito de morder lábios e bochechas, o qual resulta, então, em um processo inflamatório crônico da mucosa e hiperplasia das células locais.

Para Figueiredo CVO, et al. (2019), durante a infância e adolescência, não só a presença de biofilme dental resultante da má higiene senão a disposição dos dentes sobre a arcada dentária e o uso de aparelhos ortodônticos fixos ou removíveis são os principais fatores que culminam a formação da HFI. Já na fase adulta e na velhice, o principal fator relatado está relacionado ao uso de próteses orais mal adaptadas com a finalidade de devolver a estética e função do aparelho mastigatório, resultando na formação de lesões de origem inflamatória sobre a mucosa oral.

Para o diagnóstico, é de extrema importância a realização da biópsia, visto que é por meio de tal procedimento que se realiza a diferenciação da hiperplasia fibrosa inflamatória de outros tipos de patologias que acometem a cavidade oral. Em relação ao tratamento, o procedimento terapêutico mais indicado para a HFI é a remoção cirúrgica da lesão, com intuito de promover sua remoção total, proporcionando, portanto, uma correta cicatrização do tecido e minimização do risco de recorrência (BLOCHOWIAK K, 2015).

Por meio da realização de exame histopatológico, a lesão é caracterizada pela superprodução de tecido conjuntivo fibroso, além da presença de inúmeras células de fibroblastos e fibras colágenas, envoltas por epitélio malpighiano típico com acantose. No tecido epitelial, há o espessamento da camada de queratina (hiperqueratose). Ademais, linfócitos e células plasmáticas, como leucócitos polimorfóides e poucos vasos sanguíneos, dão origem ao infiltrado inflamatório crônico presente neste tipo de lesão. Contudo, o estado do 
tecido conjuntivo varia de acordo com o estágio em que a lesão se apresenta, uma vez que há presença de tecido de granulação em lesões jovens e do tecido conjuntivo denso e fibroso em lesões mais antigas (MATEUS OTMV, 2017).

Sendo assim, o presente trabalho teve como objetivo relatar a realização da remoção de uma lesão de hiperplasia fibrosa inflamatória presente na mucosa jugal com acometimento da papila parotídea de um paciente encaminhado à Clínica de Estomatologia da Universidade de Marília (UNIMAR).

\section{RELATO DE CASO}

Paciente C.A.C.C., sexo masculino, 56 anos de idade, leucorderma, compareceu à Clínica de Estomatologia da Faculdade de Odontologia da Universidade de Marília - UNIMAR, queixando-se da presença de uma lesão em sua mucosa jugal, próxima à região do dente 17 (segundo molar superior direito), por aproximadamente 20 anos.

Durante a realização da anamnese, o paciente relatou não ser tabagista e não fazer consumo de bebidas alcoólicas. Verificou-se que ele sofria de bruxismo, hábito parafuncional caracterizado pelo ranger e apertamento dos dentes durante o sono.

Ao exame clínico, constatou-se que a lesão possuía caráter nodular, localizada na região de papila parotídea, de consistência flácida, superfície lisa, coloração semelhante à mucosa, bem vascularizada, de inserção pediculada, ocasionalmente sintomática e com 1 centímetro de comprimento em sua maior extensão. Não havia sintomatologia dolorosa ao toque no local. Entretanto, episódios frequentes de mordiscamento sobre a lesão resultaram na presença de dor no paciente (Figura 1A).

Com isso, por meio dos dados obtidos pelo exame clínico e informações fornecidas pelo paciente durante anamnese, verificou-se que o tratamento adequado para a lesão consistia na realização de sua remoção cirúrgica completa, através da biopsia excisional.

Iniciou-se o procedimento com a realização da técnica anestésica. Para isso, foi aplicado anestésico (Mepisv 3\% - Nova DFL) próximo ao local em que a lesão estava inserida, utilizando agulha curta (Agulha Gengival Selekt Curta 30G - Kulzer), com objetivo de dessensibilizar o nervo bucal, responsável por inervar a região a ser operada. Durante tal ato, teve-se que atentar ao fato de que uma vez que o anestésico penetrasse no interior da lesão, os resultados obtidos por meio da biópsia poderiam ser comprometidos, tendo, então, que ser injetado cuidadosamente para que não obtivesse tal agravo.

Em seguida, com a finalidade de se obter melhor visualização e manipulação da lesão, realizou-se o transpasse desta com um fio de sutura (Fio de Sutura de Seda 4-0 1,7 cm Shalon), permitindo, então, sua distensão e movimentação, obtendo acesso facilitado à base.

Desse modo, tornou-se possível a realização inicial do rompimento da mucosa superficial do tecido hiperplásico, respeitando os planos de construção corpórea dos tecidos subjacentes à mucosa jugal, com auxílio de uma lâmina de bisturi (Lâmina de Bisturi de Aço Carbono 15C Swann-Morton). Houve a separação delicada dos tecidos componentes da mucosa jugal por meio de divulsão da região de base da lesão. A técnica contou com a introdução da tesoura íris de ponta reta, que penetrava de modo fechado na lesão e era retira em sua abertura, visando estabelecer a manutenção e proteção das estruturas presentes no interior da hiperplasia fibrosa inflamatória, em especial, o cuidado com o ducto parotídeo da glândula parótida.

Após adequada observação do ducto presente no interior da massa hiperplásica, foi realizado um ponto simples com a finalidade de evitar que o ducto parotídeo fosse suturado junto à massa de tecido da mucosa jugal, dado que este deveria permanecer em contato com o vestíbulo bucal para que a saliva produzida pela glândula parótida pudesse continuar sendo secretada de modo adequado. Assim, a remoção da lesão por completo da mucosa jugal tornou-se possível por meio da utilização da lâmina de bisturi (Lâmina de Bisturi de Aço Carbono 15C Swann-Morton) acoplada ao cabo de bisturi №3. Elaborou-se, então, a execução de um ponto simples para aproximação dos tecidos lesados durante o ato cirúrgico (Figura 1B e Figura 1C). 
Figura 1 - Fotografias do exame clínico e procedimentos cirúrgicos.

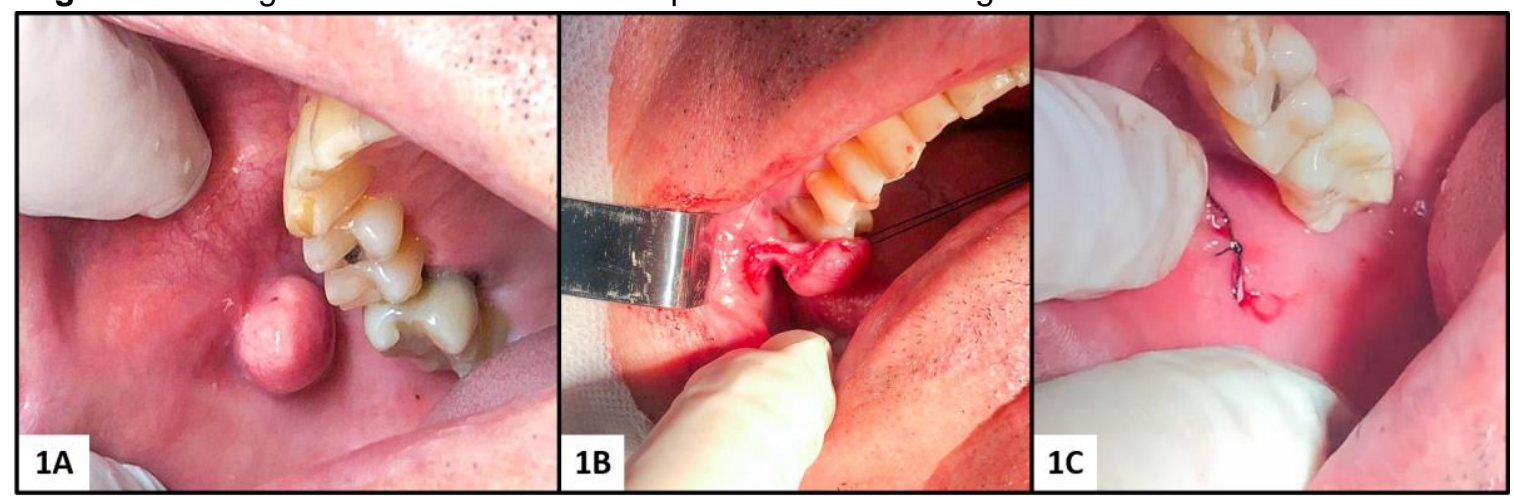

Legenda: 1A) Aspecto inicial da lesão de hiperplasia fibrosa inflamatória localizada sobre a papila parotídea. 1B) Processo de exérese da lesão, evidenciando o ducto parotídeo. 1C) Confecção de sutura simples em mucosa jugal, com finalidade de preservação do ducto parotídeo na cavidade oral.

Fonte: Canali LGM, et al., 2019.

Após remoção da lesão, esta foi inserida em um recipiente de vidro em solução formol a $10 \%$ e enviada ao exame anatomopatológico. As lâminas para análise foram confeccionadas no Laboratório de Histologia da UNIMAR e submetidas ao laboratório de diagnóstico, para que o resultado pudesse ser fornecido por um médico patologista (Figura 2A e Figura 2B).

Figura 2 - Fotografias da lesão de hiperplasia fibrosa inflamatória após remoção cirúrgica.

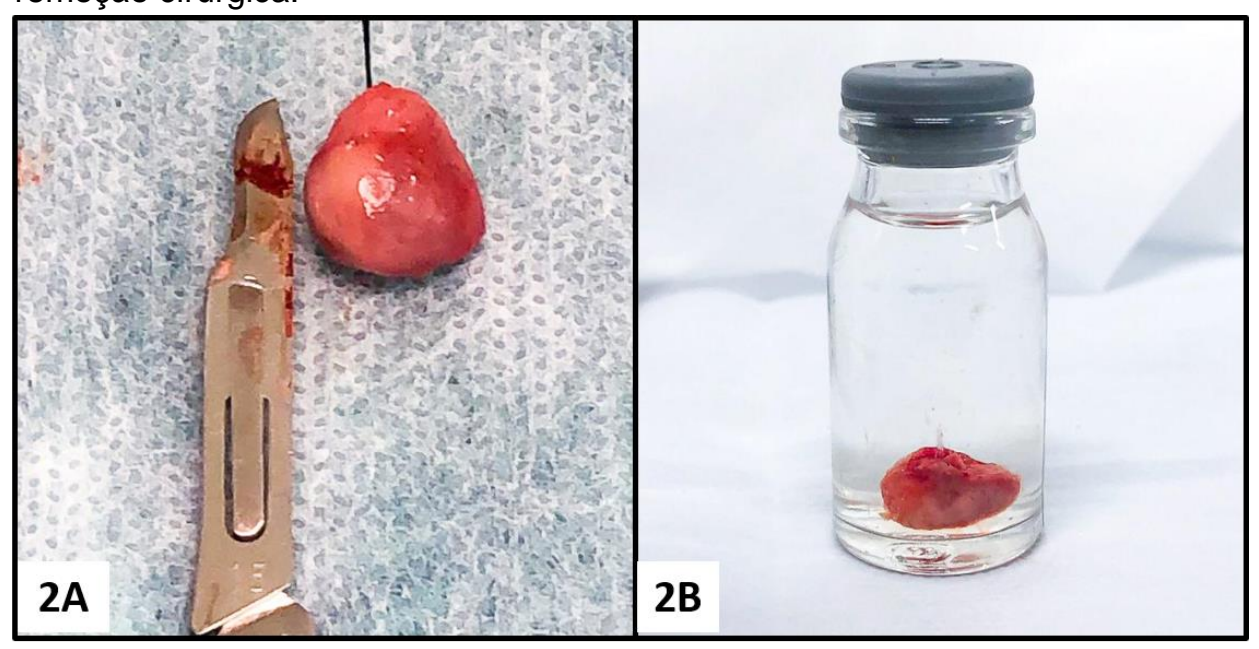

Legenda: 2A) Lesão de hiperplasia fibrosa inflamatória ao lado de lâmina de bisturi. 2B) Lesão de hiperplasia fibrosa inflamatória em solução formol a 10\%.

Fonte: Canali LGM, et al., 2019.

Após exérese da lesão em mucosa jugal, ela pôde ser visualizada macroscopicamente como um tecido irregular, medindo $1,3 \times 1,0 \mathrm{~cm}$, de coloração esbranquiçada e de consistência fibroelástica.

A partir do encaminhamento da lesão hiperplásica ao exame anatomopatológico, obteve-se suas características histológicas. Verificou-se, então, que a hiperplasia fibrosa inflamatória se baseia em uma massa nodular sólida devido à densidade do tecido conjuntivo responsável por compor a lesão. Além disso, observa-se, também, a presença de colágeno e vasos sanguíneos, característicos da formação de uma lesão cicatricial resultante dos estímulos crônicos e de baixa intensidade produzidos sobre a mucosa de revestimento. 
Em relação aos fibroblastos que compõem a lesão, estes possuem conformação estreita e alongada, dispostos paralelamente uns aos outros, além de densos e ondulados, fixos entre si. Por fim, os cortes histológicos mostraram mucosa revestida por epitélio malphigiano típico com acantose, sob o qual há proliferação de tecido conjuntivo fibroso e leve infiltrado inflamatório e, epitélio estratificado pavimentoso na luz do ducto parotídeo, localizado internamente à lesão de hiperplasia fibrosa inflamatória (Figura 3A e Figura 3B).

Figura 3 - Resultados obtidos com realização do exame anatomopatológico e lâminas histológicas.

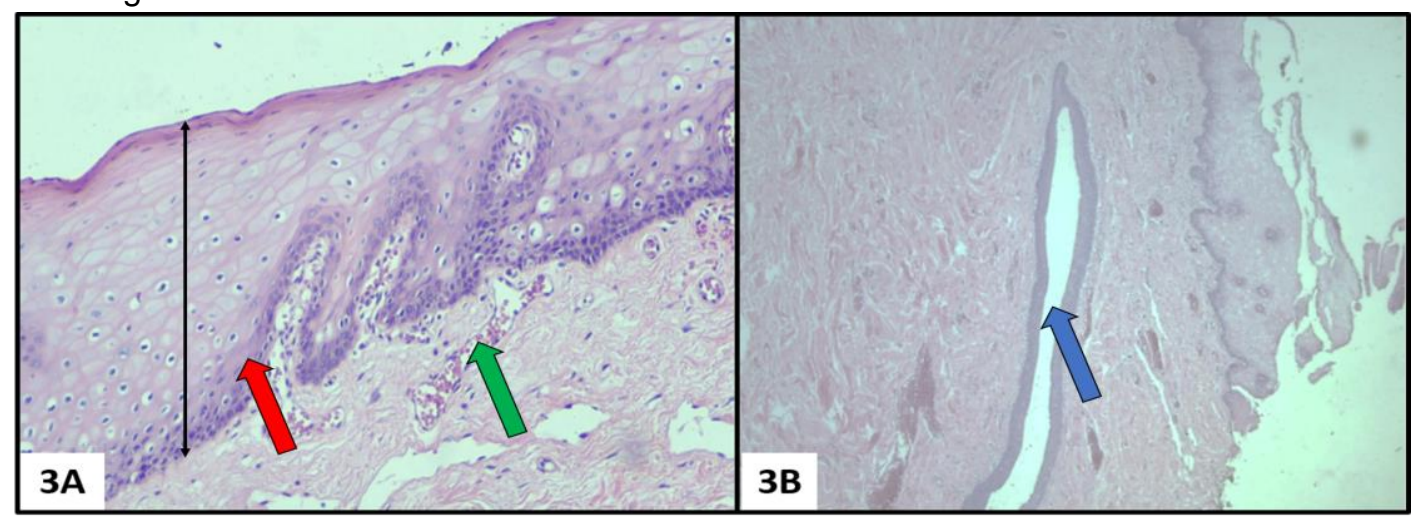

Legenda: 3A) Corte histológico evidenciando mucosa oral revestida por epitélio escamoso típico com acantose, sob o qual houve proliferação de tecido conjuntivo fibroso e leve infiltrado inflamatório linfocitário. 3B) Epitélio estratificado pavimentoso na luz do ducto parotídeo, localizado internamente à lesão de hiperplasia fibrosa inflamatória.

Fonte: Canali LGM, et al., 2019.

O paciente relatado no caso clínico foi acompanhado por um período de seis meses, e, então, verificouse, durante as consultas de retorno, uma cicatrização adequada da mucosa jugal na região em que foi realizada a excisão cirúrgica da lesão de hiperplasia fibrosa inflamatória. Além disso, o ducto parotídeo manteve-se estável, secretando adequadamente o fluido salivar na cavidade oral, garantindo, assim, o sucesso da técnica terapêutica fornecida ao paciente.

\section{DISCUSSÃO}

Considerou-se de importância a realização deste trabalho, uma vez que o tratamento da hiperplasia fibrosa inflamatória deve estar baseado em seu correto diagnóstico, havendo necessidade de descartar as demais patologias capazes de acometimento da cavidade oral e que se assemelham clinicamente com ela, tais como granuloma piogênico oral, fibroma de células gigantes, fibroma ossificante periférico e lesões periféricas de células gigantes. Análises literárias revelam que a hiperplasia fibrosa inflamatória se mostrou a mais predominante entre as lesões diagnosticadas na cavidade oral (SRIVASTAVA KC, 2019; DUTRA KL, et al., 2018; GANDHI B, 2016).

Para realização do diagnóstico, faz-se de extrema importância o exame clínico do paciente, o qual deve ser constituído pela anamnese, pelo exame físico e pelos exames complementares. Durante o procedimento cirúrgico relatado no caso, a biopsia excisional mostrou-se um importante fator constituinte como exame complementar, uma vez que possibilitou o encaminhamento da lesão para o exame anatomopatológico, com finalidade de aquisição de um correto diagnóstico e obtenção de um resultado de alta fidedignidade (MARTINS JC, et al., 2017).

Assim, na grande maioria dos casos diagnosticados como HFI, a principal opção terapêutica de escolha dos profissionais baseia-se na remoção cirúrgica da lesão pela técnica convencional com uso de bisturi, 
para realização da biopsia excisional. Sua vantagem em relação ao tratamento com utilização de lasers evidencia-se pela ausência de necessidade de treinamento intensivo do operador, baixo custo da técnica, além dos diferentes parâmetros relatados na literatura que dificultam a realização de tratamentos alternativos (JESUS AO, 2018; CANUTO TSR, 2017).

Não somente, no que diz respeito ao local no qual a lesão for constatada, a gengiva e a crista alveolar baseiam-se nos locais anatômicos em que a mesma prevalece, seguidas pela mucosa jugal ao longo da linha oclusal, dos lábios e da língua. Tal achado vai de encontro com caso clínico relatado, visto que a lesão acometeu a mucosa jugal, sobre região de papila parotídea, devido ao hábito de mordiscamento apresentado pelo paciente, submetendo o tecido a fatores irritantes crônicos (MATURANA-RAMíREZ D, et al., 2015; FARYNOWSKA J, et al. 2018).

Ademais, pôde-se constatar, também, que, em relação ao sexo de acometimento da hiperplasia fibrosa inflamatória, as mulheres mostraram-se as mais predominantes no desenvolvimento da lesão, devido à maior busca por métodos reabilitadores capazes de devolver a estética do órgão dental, tal como uso de próteses, baseando-se em um dos principais fatores etiológicos responsáveis pela formação da $\mathrm{HFI}$. Tal achado difere do sexo do paciente apresentado no relato de caso (MARTINS JC, et al., 2017; FARYNOWSKA, J et al., 2018).

Em relação à idade de acometimento, não puderam ser feitas grandes constatações, visto que a mesma foi diagnosticada pela literatura desde a primeira até a oitava década de vida. Entretanto, houve predileção pelos indivíduos componentes da faixa etária estabelecida como adultos e idosos, muito provavelmente devido ao uso de próteses mal adaptadas ou más condições de uso por um longo período (RAMU $S$ e RODRIGUES C, 2012).

A lesão hiperplásica apresentou-se, na grande maioria dos casos, de tamanho pequeno, variando de 0,5 a $2 \mathrm{~cm}$ em sua maior extensão. Tal achado clínico vai de encontro ao caráter da lesão relatada no trabalho, uma vez que esta possui $1,3 \mathrm{~cm}$ de comprimento em sua maior extensão (DUTRA KL, et al., 2018; CANUTO TSR, 2017).

Com base em análises realizadas mediante ao caso clínico relatado anteriormente, pôde ser concluído que lesões com caráter hiperplásico inflamatório ocorrem com determinada frequência em consultórios odontológicos, uma vez que sua etiologia é baseada em fatores extremamente comuns, tais como uso de próteses inadequadas, aparelhos dentários e traumas crônicos de baixa intensidade. Posto isso, cabe ao profissional da área a capacidade de fornecer um diagnóstico inicial adequado, de modo a descartar a possibilidade de lesão maligna, além de promover a remoção total da lesão e cessar sua causa de formação, permitindo, assim, melhor qualidade de vida ao paciente.

\section{REFERÊNCIAS}

1. BINITA G, et al. Reactive Lesions Of Oral Cavity. Natl J Integr Res Med, $2016 ; 7$ (4): 154-157.

2. BLOCHOWIAK K. Selected applications of Er:YAG and $\mathrm{CO}_{2}$ lasers for treatment of benign neoplasms and tumorous lesions in the mouth. Postepy Dermatol Alergol, 2015; 32(5): 337-343.

3. CANUTO TSR. Avaliação comparativa do uso do laser de Nd:YAG ou cirurgia convencional no tratamento de hiperplasia fibrosa inflamatoria. Dissertação (Mestrado em Odontologia) - Centro de Ciências da Saúde. Universidade Federal de Pernambuco, Recife, 2017; 85 p.

4. DUTRA KL, et al. Incidence of reactive hyperplastic lesions in the oral cavity: a 10 year retrospective study in Santa Catarina, Brazil. Braz J Otorhinolaryngol. 2019; 85:399-407.

5. FARYNOWSKA J, et al. Retrospective analysis of reactive hyperplastic lesions in the oral cavity. European Journal of Clinical and Experimental Medicine 2018; 16 (2): 92-96.

6. FIGUEIREDO CVO, et al. Inflammatory fibrous hyperplasia: a rare case in a child. RGO, Rev Gaúch Odontol. 2019; 67.

7. JESUS AO. Comparação da eficácia e segurança do laser cirúrgico de diodo com o eletrocautério no tratamento da hiperplasia fibrosa inflamatoria. Dissertação (Mestrado em Odontologia) - Colegiado de Pós-Graduação em Odontologia. Faculdade de Odontologia da Universidade Federal de Minas Gerais, Belo Horizonte, 2018; 94 p. 
8. MARTINS JC, et al. Prevalence of oral lesions diagnosed at the ULBRA Canoas of Dental Diagnosis Service. Stomatos, Vol. 23, № 44, Jan./Jun. 2017.

9. MATEUS OTMV. Expressão imunoistoquímica do FOXPE3+ em lesões de líquen plano bucal, cutâneo e hiperplasia fibrosa inflamatoria. Dissertação (Mestrado em Odontologia) - Programa de pós-graduação em Clínicas Odontológicas. Faculdade de Odontologia da Universidade Federal do Mato Grosso do Sul, Campo Grande, 2017; $77 \mathrm{p}$.

10. MATURANA-RAMÍREZ A, et al. A retrospective analysis of reactive hyperplastic lesions of the oral cavity: study of 1149 cases diagnosed between 2000 and 2011, Chile. Acta Odontol Latinoam, 28 (2015), pp. 103-107.

11. MENDES JK, et al. Processo Proliferativo Não Neoplásico: Granuloma Piogênico. Revista de Odontologia Contemporânea, v. 3, n. 1 Supl 2, p. 27, 16 dez. 2019.

12. RAMU S, RODRIGUES C. Reactive hyperplastic lesions of the gums: a retrospective study of 260 cases. World J Dent, 2012; 3 (2): 126-130.

13. SRIVASTAVA KC. Constellation of Oral Inflammatory Hyperplastic Lesions: Case Series with Review of Literature. Int J Med Res Health Sci 2019, 8(4): 101-106. 